\title{
Prolongations of tensor fields and connections to tangent bundles I \\ - General theory -
}

\author{
By Kentaro YANO*) and Shoshichi KoBAYASHI**)
}

(Received Dec. 13, 1965)

\section{Introduction and notations.}

The purpose of this paper is to define a natural derivational mapping (called the complete lift ${ }^{1)}$ ) of the algebra $\mathscr{I}(M)$ of tensor fields of a manifold $M$ into the algebra $\mathscr{I}(T(M))$ of tensor fields of the tangent bundle $T(M)$ of $M$, to associate with each affine connection $\nabla$ of $M$ an affine connection $\nabla^{c}$ (called the complete lift of $\nabla$ ) of $T(M)$ in a natural way and to derive basic formulas and properties of the complete lift. To define the notion of complete lift, we introduce also that of vertical lift and transvection as well as a more familiar notion of Lie derivation. The notions of complete lift and vertical lift have been already defined for tensor fields of special kinds by several authors, [5], [7], [8], [9], [14], [15].

Using the notion of complete lift we shall show that such familiar $G$ structures as a pseudo-Riemannian structure, an almost complex structure and a symplectic structure on $M$ induce similar structures on the tangent bundle $T(M)$. An unexpected but perhaps more interesting result is that each pseudo-Riemannian (resp. affine) symmetric space structure on $M$ induces a pseudo-Riemannian (resp. affine) symmetric space structure on $T(M)$. This suggests us a method of producing a large class of affine symmetric spaces. Let $A$ be a local algebra of the form $A=R+I$ where $R$ is the field of real numbers and $I$ is the maximal ideal of $A$ such that $\operatorname{dim} I<\infty$ and $I^{k}=0$ for some $k$. Weil has shown [10] that such an algebra $A$ defines a fibre bundle $A(M)$ over $M$, generalizing the construction of the tangent bundle $T(M)$ ). (If $\operatorname{dim} I=1$ and $I^{2}=0$, then $A(M)$ is nothing but the tangent bundle $T(M)$ of $M$.) A successful generalization of our theory to $A(M)$ would furnish a useful tool for the differential geometry of higher order contact and yield a large number of affine symmetric spaces.

*) Supported by NSF Grant GP-3990.

**) Sloan Fellow, partially supported by NSF Grant GP-3982.

1) Perhaps, "natural lift" is more appropriate. But in conformity with other authors, we use the term "complete lift". 


\section{Notations}

We shall generally follow notations and terminologies of [3]; in particular, components of curvature tensors are written in the same as in [3] including signs. We list below notations used often in this paper.

1. $T(M)=\bigcup_{x \in M} T_{x}(M)$ is the tangent bundle over a manifold $M$ with projection $\pi$. Similarly, $T^{*}(M)$ denotes the cotangent bundle over $M$.

2. $\mathscr{T}_{s}^{r}(M)$ is the space of tensor fields of type $(r, s)$, i. e., contravariant degree $r$ and covariant degree $s$, on $M$. An element of $\mathscr{I}_{0}^{0}(M)$ is a function and is denoted often by $f$. An element of $\mathscr{I}_{0}^{1}(M)$ is a vector field and is often denoted by $X$ or $Y$. An element of $\mathscr{I}_{1}^{0}(M)$ is a 1 -form and is often denoted by $\omega$.

3. $\mathscr{I}(M)=\sum_{r, s} \mathscr{I}_{s}^{r}(M)=\mathscr{I}^{*}(M) \otimes \mathscr{I}_{*}(M)$, where $\mathscr{I}^{*}(M)=\sum_{r} \mathscr{I}_{0}^{r}(M)$ and $\mathscr{I}_{*}(M)$ $=\sum_{s} \mathscr{I}_{s}^{0}(M)$

4. An affine connection is often denoted by its covariant differentiation symbol $\nabla$.

5. Given a local coordinate system $x^{1}, \cdots, x^{n}$ we denote by $x^{1}, \cdots, x^{n}$. $y^{1}, \cdots, y^{n}$ the local coordinate system in $T(M)$ induced as follows:

If $\bar{x}=\sum b^{i}\left(\partial / \partial x^{i}\right) \in T_{x}(M)$ and $x$ is a point with coordinates $a^{1}, \cdots, a^{n}$ with respect to $x^{1}, \cdots, x^{n}$, then $\bar{x}$ has coordinates $a^{1}, \cdots, a^{n}, b^{1}, \cdots, b^{n}$ with respect to $x^{1}, \cdots, x^{n}, y^{1}, \cdots, y^{n}$.

6. The so-called Einstein's summation convention is used.

\section{Lie derivations and transvections.}

Let $X$ be a vector field on $M$. The Lie derivation $\mathcal{L}_{X}$ with respect to $X$ is a linear endomorphism of $\mathscr{I}(M)$ characterized by the following properties:

$(\mathcal{L} .1) \quad \mathcal{L}_{X}(S \otimes T)=\left(\mathcal{L}_{X} S\right) \otimes T+S \otimes\left(\mathcal{L}_{X} T\right) \quad$ for $\quad S, T \in \mathscr{I}(M) ;$

$(\mathcal{L} .2) \quad \mathcal{L}_{X} f=X f \quad$ for $\quad f \in \mathscr{I}_{0}^{0}(M)$;

$(\mathcal{L} .3) \quad \mathcal{L}_{X} d f=d \mathcal{L}_{X} f \quad$ for $\quad f \in \mathscr{I}_{0}^{0}(M)$;

$(\mathcal{L} .4) \quad \mathcal{L}_{X} Y=[X, Y] \quad$ for $\quad Y \in \mathscr{I}_{0}^{1}(M)$.

It follows that $\mathcal{L}_{X}$ possesses also the following properties:

$(\mathcal{L} .5) \quad \mathcal{L}_{X}$ is type preserving, i. e., $\mathcal{L}_{X}\left(\mathscr{I}_{s}^{r}(M)\right) \subset \mathscr{I}_{s}^{r}(M)$;

$\left(\mathcal{L}\right.$.6) $\mathcal{L}_{X}$ commutes with every contraction of a tensor field.

For the preceding and other properties of $\mathcal{L}_{X}$, see [3] and [11].

The skew symmetric transvection $c_{X}$ by $X$ is a linear endomorphism of $\mathscr{I}(M)$ characterized by the following properties: 
$(\ell .1)$

$$
\begin{aligned}
& \iota_{X}(S \otimes T)=\left(\iota_{X} S\right) \otimes T+(-1)^{q} S \otimes\left(\iota_{X} T\right) \\
& \quad \text { for } S \in \mathscr{I}_{q}^{p}(M) \text { and } T \in \mathscr{I}(M) ; \\
& \iota_{X} f=0 \quad \text { for } \quad f \in \mathscr{I}_{0}^{0}(M) ; \\
& \iota_{X} d f=\mathcal{L}_{X} f=X f \quad \text { for } \quad f \in \mathscr{I}_{0}^{0}(M): \\
& \iota_{X} Y=0 \quad \text { for } \quad Y \in \mathscr{I}_{0}^{1}(M) .
\end{aligned}
$$

It follows that $c_{X}$ possesses also the following properties:

$$
\iota_{X}\left(\mathscr{I}_{s}^{r}(M)\right) \subset \mathscr{I}_{s-1}^{r}(M) \text {, in particular, } \iota_{X}\left(\mathscr{I}_{0}^{r}(M)\right)=0 \text { : }
$$

$$
\iota_{X} \circ \iota_{X}=0 \text {. }
$$

When applied to differential forms, $\iota_{X}$ is often called the interior product, [3]. From (८.1) we obtain also

(c.7) $\iota_{X} K=0$ if $K \in \mathscr{I}_{s}^{0}(M)$ is a symmetric covariant tensor field.

The symmetric transvection $\sigma_{X}$ by $X$ is a linear endomorphism of $\mathscr{I}(M)$ characterized by the following properties:

$$
\begin{array}{ll}
(\sigma .1) & \sigma_{X}(S \otimes T)=\left(\sigma_{X} S\right) \otimes T+S \otimes\left(\sigma_{X} T\right) \quad \text { for } \quad S, T \in \mathscr{I}(M): \\
(\sigma .2) & \sigma_{X} f=0 \quad \text { for } \quad f \in \mathscr{I}_{0}^{0}(M): \\
(\sigma .3) & \sigma_{X} d f=\mathcal{L}_{X} f=X f \quad \text { for } \quad f \in \mathscr{I}_{0}^{0}(M)
\end{array}
$$$$
\text { ( } \sigma .4) \quad \sigma_{X} Y=0 \quad \text { for } \quad Y \in \mathscr{I}_{0}^{1}(M) \text {. }
$$

It follows that $\sigma_{X}$ possesses also the following properties:

$(\sigma .5) \quad \sigma_{X}\left(\mathscr{I}_{s}^{r}(M)\right) \subset \mathscr{I}_{s^{-1}}^{r}(M)$, in particular, $\sigma_{X}\left(\mathscr{I}_{0}^{r}(M)\right)=0$;

( $\sigma .6) \quad \sigma_{X} \circ \sigma_{Y}=\sigma_{Y} \circ \sigma_{X}$.

In contrast to (c.7), we have

( $\sigma .7) \quad \sigma_{X} K=0$ if $K \in \mathscr{I}_{s}^{0}(M)$ is a skew-symmetric covariant tensor field.

We now fix a positive integer $k$. Then, for $s \geqq k$, every vector field $X$ defines a linear mapping $\gamma_{X}: \mathscr{I}_{s}^{r}(M) \rightarrow \mathscr{I}_{s-1}^{r}(M)$ such that

$$
\gamma_{X}\left(S \otimes \omega_{1} \otimes \cdots \otimes \omega_{k} \otimes \cdots \otimes \omega_{s}\right)=S \otimes \omega_{1} \otimes \cdots \otimes \omega_{k}(X) \otimes \cdots \otimes \omega_{s},
$$

where $S \in \mathscr{I}_{0}^{r}(M)$ and $\omega_{i} \in \mathscr{I}_{1}^{0}(M)$ for $i=1, \cdots, s$.

In terms of a local coordinate system $x^{1}, \cdots, x^{n}$ of $M$, let $K_{j_{1} \cdots j_{s}}^{i_{1} \ldots i r}$ be the components of a tensor field $K \in \mathscr{T}_{s}^{r}(M)$ and $\xi^{i}$ the components of $X$. Then $\gamma_{X} K$ is the tensor field of type $(r, s-1)$ with components $K_{j_{1} \cdots j_{k} \cdots j_{s}}^{i_{1} \cdots i r} \xi^{j_{k}}$.

If $s=1$ so that $k=1$ necessarily, then $\gamma_{X}$ coincides with $\iota_{X}$ and $\sigma_{X}$. Considering $\gamma_{X}$ for all $k, 1 \leqq k \leqq s$, it is easy to express both $\iota_{X}$ and $\sigma_{X}$ by means of $\gamma_{x}$. Since $\iota_{x}, \sigma_{X}$ and $\gamma_{x}$ behave in a similar manner, they will be denoted by $\alpha_{X}$ when the distinction is not necessary.

Proposition 2.1. For $X, Y \in \mathscr{I}_{0}^{1}(M)$ we have 
(1) $\left[\mathcal{L}_{X}, \mathcal{L}_{Y}\right]=\mathcal{L}_{[X, Y]}$;

(2) $\left[\mathcal{L}_{X}, \alpha_{Y}\right]=\alpha_{[X, Y]}$;

(3) $\mathcal{L}_{X} \omega=d \circ \iota_{X} \omega+\iota_{X} \circ d \omega$ for any differential form $\omega$.

Proof. Since $\mathscr{I}(M)$ is generated by $f \in \mathscr{I}_{0}^{0}(M), d f \in \mathscr{I}_{1}^{0}(M)$ and $Z \in \mathscr{I}_{0}^{1}(M)$, it is sufficient to prove (1) and (2) when the both sides are applied to $f, d f$ and $Z$. The verification in the three special cases are straightforward and are left to the reader. Similarly, it is sufficient to verify (3) in the cases where $\omega=f$ and $\omega=d f$. For (1) and (3) we refer the reader also to [3; p. 32 and p. 35].

Q.E.D.

\section{Vertical lifts.}

Let $\bar{x} \in T_{x}(M)$. The projection $\pi: T(M) \rightarrow M$ induces a surjective linear mapping $\pi_{*}: T_{\bar{x}}(T(M)) \rightarrow T_{x}(M)$, called the differential of $\pi$ at $\bar{x}$. Its dual mapping $\pi^{*}: T_{x}^{*}(M) \rightarrow T_{x}^{*}(T(M))$ is injective. Clearly, $\pi^{*}$ can be extended to a unique isomorphism of the covariant tensor algebra $\mathscr{T}_{*}(x)$ at $x$ into the covariant tensor algebra $\mathscr{I}_{*}(\bar{x})$ at $\bar{x}$. This gives rise to an isomorphism, called the vertical lift, of the algebra $\mathscr{T}_{*}(M)$ of covariant tensor fields of $M$ into the algebra $\mathscr{T}_{*}(T(M))$ of covariant tensor fields of $T(M)$. For a covariant tensor field $K \in \mathscr{I}_{*}(M)$, its vertical lift will be denoted by $K^{\nu}$. The following is immediate from the definition.

Proposition 3.1. (1) $f^{v}=f \circ \pi$ for $f \in \mathscr{I}_{0}^{0}(M)$;

(2) For $K \in \mathscr{I}_{q}^{0}(M)$ considered as a multilinear mapping $T_{x}(M) \times \cdots \times T_{x}(M)$ $\rightarrow R^{n}$, its vertical lift $K^{v}: T-(T(M)) \times \cdots \times T_{\bar{x}}(T(M)) \rightarrow R^{n}$ satisfies

$$
K^{v}\left(\tilde{X}_{1}, \cdots, \tilde{X}_{q}\right)=K\left(\pi_{*} \tilde{X}_{1}, \cdots, \pi_{*} \tilde{X}_{q}\right) \quad \text { for } \quad \tilde{X}_{1} \in T_{\bar{r}}(T(M)) ;
$$

(3) The vertical lift maps the algebra $\mathscr{D}(M)$ of differential forms of $M$ isomorphically into the algebra $\mathscr{D}(T(M))$ of differential forms of $T(M)$.

The vertical lift $\mathscr{D}(M) \rightarrow \mathscr{D}(T(M))$ is usually denoted by $\pi^{*}$.

To introduce the notion of vertical lift to the algebra of contravariant tensor fields, we define two linear mappings $c$ and $\sigma$ of $\mathscr{I}_{*}(M)$ into $\mathscr{I}_{*}(T(M)$ ) which are similar to $\iota_{X}$ and $\sigma_{x}$. The mapping $\iota: \mathscr{I}_{*}(M) \rightarrow \mathscr{I}_{*}(T(M))$ is a linear mapping characterized by

$$
\begin{gathered}
\iota(S \otimes T)=(\iota S) \otimes T^{v}+(-1)^{q} S^{v} \otimes(\iota T) \\
\text { for } S \in \mathscr{I}_{q}^{0}(M) \text { and } T \in \mathscr{I}_{*}(M) ; \\
\iota f=0 \quad \text { for } \quad f \in \mathscr{I}_{0}^{0}(M) ; \\
\iota(d f)=d f \quad \text { for } \quad t \in \mathscr{I}_{0}^{0}(M),
\end{gathered}
$$

where $d f$ on the right hand side is considered as a function on $T(M)$. 
The mapping $\sigma: \mathscr{T}_{*}(M) \rightarrow \mathscr{I}_{*}(T(M))$ is a linear mapping characterized by $(\sigma .1)^{\prime} \quad \sigma(S \otimes T)=(\sigma S) \otimes T^{v}+S^{v} \otimes(\sigma T) \quad$ for $\quad S, T \in \mathscr{I}_{*}(M)$;

$(\sigma .3)^{\prime}$

$$
\begin{gathered}
\sigma f=0 \quad \text { for } \quad f \in \mathcal{I}_{0}^{0}(M) ; \\
\sigma(d f)=d f \quad \text { for } \quad f \in \mathcal{I}_{0}^{0}(M),
\end{gathered}
$$

where $d f$ on the right hand side is considered as a function on $T(M)$.

Later these mappings $c$ and $\sigma$ will be extended to linear mappings of $\mathscr{I}(M)$ into $\mathscr{I}(T(M))$. We note that if

$$
\omega=f_{i} d x^{2}
$$

in terms of a local coordinate system $x^{1}, \cdots, x^{n}$ of $M$, then

$$
c(\omega)=\sigma(\omega)=f_{i} y^{2}
$$

in terms of the induced local coordinate system $x^{1}, \cdots, x^{n}, y^{1}, \cdots, y^{n}$ of $T(M)$.

As a first step to extend the vertical lift to the algebra $\mathscr{I}(M)$, we define a vertical lift $X^{v}$ of a vector field $X$ of $M$. It is a vector field on $T(M)$ characterized by

$$
X^{v}(\iota(d f))=(X f)^{v} \quad \text { for } \quad f \in \mathscr{I}_{0}^{0}(M) .
$$

In terms of a local coordinate system $x^{1}, \cdots, x^{n}$ of $M$, if

$$
X=\xi^{i} \frac{\partial}{\partial x^{i}} \quad \text { and } \quad d f=f_{i} d x^{\imath}
$$

then

$$
X^{v}=\xi^{i} \frac{\partial}{\partial y^{i}},
$$

in terms of the induced local coordinate system $x^{1}, \cdots, x^{n}, y^{1}, \cdots, y^{n}$ of $T(M)$. This proves the uniqueness as well as the existence of $X^{v}$ satisfying (*).

By $(*)$ the vertical lift $\mathscr{I}_{0}^{1}(M) \rightarrow \mathscr{I}_{0}^{1}(T(M))$ is clearly injective. It should be warned however that it is not a Lie algebra homomorphism.

We extend the vertical lift $\mathscr{I}_{0}^{1}(M) \rightarrow \mathscr{I}_{0}^{1}(T(M)$ ) to a unique algebra isomorphism of $\mathscr{T}^{*}(M)$ into $\mathscr{I}^{*}(T(M))$. By tensoring the two vertical lift $\mathscr{T}_{*}(M)$ $\rightarrow I_{*}(T(M))$ and $I^{*}(M) \rightarrow I^{*}(T(M))$ we obtain an algebra isomorphism of $\mathscr{T}(M)$ into $\mathscr{I}(T(M)$ ), which is called the vertical lift. In resumé we may say that the vertical lift is a linear mapping of $\mathscr{T}(M)$ into $\mathscr{I}(T(M)$ ) characterized by the following properties:

$$
\begin{array}{cc}
(v .1) & (S \otimes T)^{v}=S^{v} \otimes T^{v} \quad \text { for } \quad S, T \in \mathscr{I}(M) ; \\
(v .2) & f^{v}=f \quad \text { for } \quad f \in \mathscr{I}_{0}^{0}(M) ; \\
(v .3) & (d f)^{v}=d\left(f^{v}\right) \text { for } f \in \mathscr{I}_{0}^{0}(M) ; \\
(v .4) & X^{v}(\ell(d f))=(X f)^{v} \quad \text { for } \quad X \in \mathscr{I}_{0}^{1}(M) \text { and } f \in \mathscr{I}_{0}^{0}(M) .
\end{array}
$$


We are now in position to extend $\iota$ and $\sigma$ to linear mappings of $\mathscr{T}(M)$ into $\mathscr{I}(T(M))$. They can be characterized by the following properties :

$$
\begin{array}{cc}
(\iota .1)^{*} & \iota(S \otimes T)=(\iota S) \otimes T^{v}+(-1)^{q} S^{v} \otimes(\iota T) \\
& \text { for } S \in \mathscr{T}_{q}^{p}(M) \quad \text { and } T \in \mathscr{I}(M) ; \\
(\iota .2)^{*} & \iota f=0 \quad \text { for } \quad f \in \mathscr{I}_{0}^{0}(M) ; \\
(\iota .3)^{*} & \iota(d f)=d f \quad \text { for } \quad f \in \mathscr{I}_{0}^{0}(M),
\end{array}
$$

where $d f$ on the right hand side is considered as a function on $T(M)$;

$(c .4)^{*}$

$$
\text { ‘ } X=0 \quad \text { for } \quad X \in \mathscr{I}_{0}^{1}(M) \text {. }
$$

It follows that

$(\iota .5)^{*}$

$$
\iota\left(\mathscr{I}_{s}^{r}(M)\right) \subset \mathscr{I}_{s-1}^{r}(T(M))
$$

Similarly,

$$
\begin{array}{ccc}
(\sigma .1)^{*} & \sigma(S \otimes T)=(\sigma S) \otimes T^{v}+S^{v} \otimes(\sigma T) \quad \text { for } & S, T \in \mathscr{I}(M) ; \\
(\sigma 2)^{*} & \sigma f=0 \quad \text { for } \quad f \in \mathscr{I}_{0}^{0}(M) ; \\
(\sigma .3)^{*} & \sigma(d f)=d f \quad \text { for } \quad f \in \mathscr{I}_{0}^{0}(M),
\end{array}
$$

where $d f$ on the right hand side is considered as a function on $T(M)$;

$(\sigma .4)^{*}$

$$
\sigma X=0 \quad \text { for } \quad X \in \mathscr{I}_{0}^{1}(M) \text {. }
$$

It follows that

$(\sigma .5)^{*}$

$$
\sigma\left(\mathscr{I}_{s}^{r}(M)\right) \subset T_{s-1}^{r}(T(M))
$$

Evidently,

and

$$
\iota=\sigma \quad \text { on } \quad \mathscr{I}_{1}^{0}(M)
$$

$$
\iota=\sigma=0 \quad \text { on } \quad \mathscr{T}^{*}(M) .
$$

As an example we mention the canonical vector field on $T(M)$; it is: defined to be $\iota I=\sigma I$, where $I \in I_{1}^{1}(M)$ is the field of identity endomorphisms. In terms of the local coordinate system $x^{1}, \cdots, x^{n}, y^{1}, \cdots, y^{n}$ of $T(M)$ induced by a local coordinate system $x^{1}, \cdots, x^{n}$ of $M$,

$$
\iota I=\sigma I=y^{2} \frac{\partial}{\partial y^{i}} .
$$

We now fix a positive integer $k$. Then in a similar manner as we defined $\gamma_{X}$ in $\S 2$, we define a linear mapping $\gamma: \mathscr{I}_{s}^{r}(M) \rightarrow \mathscr{I}_{s-1}^{r}(T(M))$ for $s \geqq k$ by

$$
\gamma\left(S \otimes \omega_{1} \otimes \cdots \otimes \omega_{k} \otimes \cdots \otimes \omega_{s}\right)=S^{V} \otimes \omega_{1}^{V} \otimes \cdots \otimes \imath\left(\omega_{k}\right) \otimes \cdots \otimes \omega_{s}^{V}
$$

where $S \in \mathscr{I}_{0}^{r}(M)$ and $\omega_{i} \in \mathscr{I}_{1}^{0}(M)$.

In terms of a local coordinate system $x^{1}, \cdots, x^{n}$ of $M$ and the induced local coordinate system $x^{1}, \cdots, x^{n}, y^{1}, \cdots, y^{n}$ of $T(M)$. 


$$
\begin{aligned}
& r\left(K_{j_{1} \cdots j_{s}}^{i_{1} \cdots i_{r}} \frac{\partial}{\partial x^{i_{1}}} \otimes \cdots \otimes \frac{\partial}{\partial x^{i_{r}}} \otimes d x^{j_{1}} \otimes \cdots \otimes d x^{\jmath^{s}}\right) \\
& \quad=K_{j_{1} \cdots j_{k} \cdots j_{s}}^{i_{1} \cdots i_{r}} y^{j k} \frac{\partial}{\partial y^{i_{1}}} \otimes \cdots \otimes \frac{\partial}{\partial y^{i_{r}}} \otimes d x^{j_{1}} \otimes \cdots \otimes \widehat{d x}^{j k} \otimes \cdots \otimes d x^{j_{s}}
\end{aligned}
$$

If $s=1$ so that $k=1$ necessarily, then $\gamma$ coincides with $c$ and $\sigma$. Considering $\gamma$ for all $k, 1 \leqq k \leqq s$, it is easy to express both $c$ and $\sigma$ by means of $\gamma$. Since $\iota, \sigma$ and $\gamma$ behave in a similar manner, they will be denoted by $\alpha$ when no distinction is necessary.

\section{Formulas on vertical lifts.}

Throughout this section, $X$ is a vector field on $M$ and $K$ is a tensor field on $M$. We recall that $\alpha_{X}$ (resp. $\alpha$ ) stands for any one of $\iota_{X}, \sigma_{X}$ and $\gamma_{X}$ (resp. ८, $\sigma$ and $\gamma$ ).

PROPOSITION 4.1.

$$
\begin{aligned}
& \mathcal{L}_{X^{v}}\left(K^{v}\right)=0 ; \\
& \alpha_{X^{v}}\left(K^{v}\right)=0 .
\end{aligned}
$$

Proof. Since the vertical lift is an algebra isomorphism of $\mathscr{T}(M)$ into $\mathscr{T}\left(T(M)\right.$ ) and since $\mathscr{I}(M)$ is generated by $f \in \mathscr{I}_{0}^{0}(M), d f \in \mathscr{I}_{1}^{0}(M)$ and $Y \in \mathscr{T}_{0}^{1}(M)$, it suffices to verify the formulas above in the special cases where $K=f$, $K=d f$ and $K=Y$. The verifications in these special cases are trivial if one writes $X=\xi^{i}\left(\partial / \partial x^{i}\right)$ and $X^{v}=\xi^{i}\left(\partial / \partial y^{i}\right)$ in terms of the local coordinate system $x^{1}, \cdots, x^{n}, y^{1}, \cdots, y^{n}$ of $T(M)$ induced by a local coordinate system $x^{1}, \cdots, x^{n}$ of $M$.

Q.E.D.

\section{PROPOSITION 4.2 .}

$$
\mathcal{L}_{X^{V}}(\alpha K)=\left(\alpha_{X} K\right)^{V} .
$$

Proof. We shall prove the formula for the case $\alpha=\sigma$. By (1) of Proposition 4.1 we have

$$
\mathcal{L}_{X} v(\sigma(S \otimes T))=\left(\mathcal{L}_{X^{v}}(\sigma S)\right) \otimes T^{v}+S^{v} \otimes\left(\mathcal{L}_{X^{v}}(\sigma T)\right) .
$$

Since the vertical lift is an algebra homomorphism, we have

$$
\left(\sigma_{X}(S \otimes T)\right)^{v}=\left(\sigma_{X} S\right)^{v} \otimes T^{v}+S^{v} \otimes\left(\sigma_{X} T\right)^{v} .
$$

Hence it suffices to prove the formula in the special cases where $K=f, K=d f$ and $K=Y$ for the same reason as in the proof of Proposition 4.1. If $K=f$ or $K=Y$, then the both sides of the formula vanish. If $K=d f$, we write $X=\xi^{i}\left(\partial / \partial x^{i}\right)$ and $d f=f_{i} d x^{i}$. Then $\sigma(d f)=f_{i} y^{i}$ and $X^{v}=\xi^{i}\left(\partial / \partial y^{i}\right)$. Hence both the left and the right hand sides are equal to $f_{i} \xi^{2}$

Q.E.D. 


\section{Complete lifts of tensor fields.}

The complete lift is a linear mapping of $\mathscr{I}(M)$ into $\mathscr{I}(T(M)$ ) characterized by the following properties. If we denote the complete lift of $K \in \mathscr{T}(M)$ by $K^{c}$, then

$$
\begin{gathered}
(S \otimes T)^{c}=S^{c} \otimes T^{v}+S^{v} \otimes T^{\circ} \quad \text { for } \quad S, T \in \mathscr{I}(M) ; \\
f^{c}=\iota(d f)=\sigma(d f) \quad \text { for } \quad f \in \mathscr{I}_{0}^{0}(M) ; \\
(d f)^{c}=d\left(f^{c}\right) \quad \text { for } \quad f \in \mathscr{I}_{0}^{0}(M) ;
\end{gathered}
$$

It follows that

$$
\text { if } K \in \mathscr{T}_{s}^{r}(M) \text {, then } K^{o} \in \mathscr{I}_{s}^{r}(T(M)) \text {. }
$$

In terms of the local coordinate system $x^{1}, \cdots, x^{n}, y^{1}, \cdots, y^{n}$ of $T(M)$ induced by a local coordinate system $x^{1}, \cdots, x^{n}$ of $M$, we obtain easily

$$
\begin{gathered}
f^{c}=f_{i} y^{i} \quad \text { where } f_{i}=\partial f / \partial x^{i} \\
(d f)^{c}=y^{i} d f_{i}+f_{i} d y^{i} \quad \text { where } f_{i}=\partial f / \partial x^{i} \\
X^{c}=\xi^{i} \frac{\partial}{\partial x^{i}}+\frac{\partial \xi^{i}}{\partial x^{j}} y^{j} \frac{\partial}{\partial y^{i}} \quad \text { where } \quad X=\xi^{i} \frac{\partial}{\partial x^{i}} .
\end{gathered}
$$

The exisence and the uniqueness of the complete lift may be proved readily from these formulas in local coordinate system.

REMARK. It is also clear from the formula above of $X^{c}$ that the set of all $X^{c}$, where $X \in \mathscr{T}_{0}^{1}(M)$, gives the whole tangent space at each point of $T(M)$ except at the zero points. The zero points of $T(M)$, i. e., the zero tangent vectors of $M$, may be identified with $M$ in a natural manner. Since $T(M)-M$ is dense in $T(M)$, a continuous mapping of $\mathscr{T}(T(M))$ into a vector space sends $\mathscr{I}\left(T(M)\right.$ ) into the zero element if it sends each $X^{0}$ into zero. This remark is useful in simplifying later the proofs of certain formulas.

Proposition 5.1. Let $X \in \mathscr{I}_{0}^{1}(M)$ and $K \in \mathscr{T}(M)$. Let $\alpha$ (resp. $\alpha_{X}$ ) stand for any one of $\iota, \sigma$ and $\gamma\left(\right.$ resp. $\iota_{X}, \sigma_{X}$ and $\left.\gamma_{X}\right)$. Then

$$
\begin{aligned}
& \mathcal{L}_{X^{c}}\left(K^{c}\right)=\left(\mathcal{L}_{X} K\right)^{c} ; \\
& \mathcal{L}_{X^{C}}\left(K^{v}\right)=\left(\mathcal{L}_{X} K\right)^{v} ; \\
& \mathcal{L}_{X^{V}}\left(K^{c}\right)=\left(\mathcal{L}_{X} K\right)^{V} ; \\
& \mathcal{L}_{X^{v}} \circ \alpha=\alpha \circ \mathcal{L}_{X} ; \\
& \alpha_{X}\left(K^{c}\right)=\left(\alpha_{X} K\right)^{c} ; \\
& \alpha_{X}\left(K^{v}\right)=\left(\alpha_{X} K\right)^{v} ; \\
& \alpha_{X}\left(K^{c}\right)=\left(\alpha_{X} K\right)^{V} .
\end{aligned}
$$


It is sufficient to verify these formulas in the special cases where $K=f$, $K=d f$ and $K=Y$. The verifications in the special cases are straightforward and are left to the reader.

\section{Complete lifts of special tensor fields.}

Let $K \in \mathscr{T}_{s}^{r}(M)$ and consider it as a multilinear mapping of $\mathscr{I}_{0}^{1}(M) \times \cdots$ $\times \mathscr{I}_{0}^{1}(M)$ ( $s$ times) into $\mathscr{I}_{0}^{r}(M)$ under the natural identification $\mathscr{I}_{s}^{r}(M)=\mathscr{I}_{0}^{r}(M)$ $\otimes \mathscr{I}_{s}^{0}(M)=\operatorname{Hom}\left(I_{0}^{s}, I_{0}^{r}\right)$. From (5) of Proposition 5.1 we obtain

PROPOSITION 6.1. If $K \in \mathscr{I}_{s}^{r}(M)$, then

$$
K^{c}\left(X_{1}^{C}, \cdots, X_{s}^{C}\right)=\left(K\left(X_{1}, \cdots, X_{s}\right)\right)^{C} \quad \text { for } \quad X_{i} \in \mathscr{I}_{0}^{1}(M) .
$$

From Proposition 6.1 and from the remark made in $\S 5$, we may conclude that if $K \in \mathscr{I}_{s}^{r}(M)$ is a symmetric (resp. skew-symmetric) multilinear mapping of $\mathscr{I}_{0}^{1}(M) \times \cdots \times \mathscr{I}_{0}^{1}(M)$ into $\mathscr{I}_{0}^{r}(M)$, then $K^{c} \in \mathscr{I}_{s}^{r}(T(M))$ is also a symmetric (resp. skew-symmetric) multilinear mapping of $\mathscr{I}_{0}^{1}(T(M)) \times \cdots \times I_{0}^{1}(T(M)$ ) into $\mathscr{I}_{0}^{r}(T(M))$. In particular, if $\varphi$ is a $p$-form on $M$, then $\varphi^{C}$ is a $p$-form on $T(M)$.

PROPOSITION 6.2. For any differential forms $\varphi$ and $\phi$ of $M$, we have

$$
\begin{gathered}
(\varphi \wedge \psi)^{C}=\varphi^{C} \wedge \phi^{V}+\varphi^{V} \wedge \phi^{C} ; \\
(d \varphi)^{C}=d\left(\varphi^{C}\right) .
\end{gathered}
$$

Proof. From Proposition 6.1 it follows that the complete lift commutes with skew-symmetrization. Hence (1) follows from (c.1). (2) follows from (1), $\begin{array}{lll}\text { (c.3) and the fact that the vertical lift also commutes with } d \text {. } & \text { Q.E.D. }\end{array}$

We shall now study the complete lift $K^{c}$ of a tensor field of type $(0,2)$ a little in detail. If we write

$$
K=K_{i j} d x^{i} \otimes d x^{j}
$$

in terms of a local coordinate system $x^{1}, \cdots, x^{n}$ of $M$, then (c.1), (c.2) and (c.3) imply that

$$
K^{C}=\frac{\partial K_{i j}}{\partial x^{k}} y^{k} d x^{i} \otimes d x^{j}+K_{i j} d y^{i} \otimes d x^{j}+K_{i j} d x^{i} \otimes d y^{j}
$$

in terms of the induced local coordinate system $x^{1}, \cdots, x^{n}, y^{1}, \cdots, y^{n}$ of $T(M)$. If we express $K$ by an $(n \times n)$ matrix $\left(K_{i j}\right)$, then $K^{C}$ may be expressed by a $(2 n \times 2 n)$ matrix :

$$
\left(\begin{array}{cc}
\frac{\partial K_{i j}}{\partial x^{k}} y^{k} & K_{i j} \\
K_{i j} & 0
\end{array}\right) .
$$

From this we may derive a number of properties of $K^{c}$. 
Proposition 6.3. Let $g$ be a pseudo-Riemannian metric on $M$. Then $g^{C}$ is a pseudo-Riemannian metric on $T(M)$ (with $n$ positive and $n$ negative signs).

PROOF. If we take a normal coordinate system, then at the origin $\partial g_{i j} / \partial x^{k}$ vanish. Our statement is now obvious from the matrix expression for $K^{c}$ given above.

Q.E.D.

REMARK. If we write $d s^{2}=g_{i j} d x^{i} d x^{j}$ for $g$, then $g^{c}$ may be written $2 g_{i j} \delta y^{i} d x^{j}$, where $\delta y^{2}=d y^{i}+\Gamma_{j k}^{i} d x^{j} y^{k}$.

Proposition 6.4. If $\varphi$ is a 2-form defining an (almost) symplectic structure on $M$, then $\varphi^{C}$ defines an (almost) symplectic structure on $T(M)$.

Proof. From the expression above for $K^{c}$, it is clear that if $\varphi$ is of maximal rank, so is $\varphi^{c}$. If $\varphi$ is closed, so is $\omega^{C}$ by Proposition 6.2.

We shall now study tensor fields of type $(1,1)$.

Proposition 6.5. Let $A, B \in \mathscr{I}_{1}^{1}(M)$ and consider them as fields of linear endomorphisms of tangent spaces of $M$. Let $I$ be the field of identity transformations of tangent spaces of $M$. Then

$$
(A \circ B)^{C}=A^{C} \circ B^{C} \text {, }
$$

$I^{C}=$ the field of identity automorphisms of tangent spaces of $T(M)$. In particular, if $P$ is a polynomial of one variable, then

$$
P\left(A^{C}\right)=(P(A))^{C} .
$$

Proof. By Proposition 6.1, we have

$$
(A \circ B)^{C}\left(X^{c}\right)=((A \circ B)(X))^{C}=(A(B(X)))^{C}=A^{C}\left(B^{c}\left(X^{c}\right)\right)=\left(A^{c} \circ B^{c}\right)\left(X^{c}\right) .
$$

By the remark made in $\S 5$ we may conclude that $(A \circ B)^{C}=A^{C} \circ B^{C}$. Similarly,

$$
I^{c}\left(X^{c}\right)=(I(X))^{c}=X^{c},
$$

and by the same remark we may conclude that $I^{C}$ is the field of identity automorphisms of tangent spaces of $T(M)$.

Q.E.D.

Let $A, B \in \mathscr{I}_{1}^{1}(M)$. Then the torsion $t_{A, B}(X, Y)$ of $A$ and $B$ is a tensor field of type $(1,2)$ defined by $(\text { cf. }[3])^{2)}$

$$
\begin{aligned}
& t_{A, B}(X, Y)=[A X, B Y]+[B X, A Y]+A B[X, Y]+B A[X, Y] \\
& \quad-A[X, B Y]-A[B X, Y]-B[X, A Y]-B[A X, Y] \text { for } X, Y \in \mathscr{I}_{0}^{1}(M) .
\end{aligned}
$$

From the definition of the torsion, Propsitions 6.1 and 6.5 and (1) of Proposition 5.1, we obtain

Proposition 6.6. Let $A, B \in \mathscr{I}_{1}^{1}(M)$. Then

2) The notion of torsion $t_{A, B}(X, Y)$ is due to Nijenhuis although only the special case $\frac{1}{2} t_{J, J}(X, Y)$ (where $J$ is an almost complex structure) is widely known as the Nijenhuis tensor. See, A. Nijenhuis, $X_{n-1}$-forming sets of eigenvectors, Indag. Math., 13 (1951), 200-212. 


$$
\left(t_{A, B}\right)^{C}=t_{A} C, B^{C}
$$

From Propositions 6.5 and 6.6 we obtain

Proposition 6.7. If $J \in \mathscr{I}_{1}^{1}(M)$ defines an almost complex structure on $M$, so does $J^{c}$ on $T(M)$. If $N_{J}=\frac{1}{2} t_{J, J}$ denotes the Nijenhuis tensor of $J,\left(N_{J}\right)^{c}$ is the Nijenhuis tensor of $J^{C}:\left(N_{J}\right)^{C}=N_{J^{C}}$.

If we write $A \in \mathscr{I}_{1}^{1}(M)$

$$
A=A_{j}^{i} \frac{\partial}{\partial x^{i}} \otimes d x^{j}
$$

in terms of a local coordinate system $x^{1}, \cdots, x^{n}$ of $M$, then (c.1-4) imply that

$$
A^{c}=A_{j}^{i} \frac{\partial}{\partial x^{i}} \otimes d x^{j}+\frac{\partial A_{j}^{i}}{\partial x^{k}} y^{k} \frac{\partial}{\partial y^{i}} \otimes d x^{j}+A_{j}^{i} \frac{\partial}{\partial y^{i}} \otimes d y^{j}
$$

in terms of the induced local coordinate system $x^{1}, \cdots, x^{n}, y^{1}, \cdots, y^{n}$ of $T(M)$. If we express $A$ by an $(n \times n)$ matrix $\left(A_{j}^{i}\right)$, then $A^{C}$ may be expressed by a $(2 n \times 2 n)$ matrix :

$$
\left(\begin{array}{cc}
A_{\jmath}^{\iota} & 0 \\
\frac{\partial A_{j}^{i}}{\partial x^{k}} y^{k} & A_{j}^{i}
\end{array}\right) .
$$

It is clear from this expression for $A^{C}$ that if $A$ is of rank $r$ at each point of $M$, then $A^{C}$ is of rank $2 r$ at each point of $T(M)$.

As another example we mention a tensor field $F \in \mathscr{T}_{1}^{1}(M)$ satisfying $F^{3}+F$ $=0$; such a structure has been studied in [12], [13]. Then $F^{C} \in \mathscr{I}_{1}^{1}(T(M)$ ) satisfies $\left(F^{C}\right)^{3}+F^{C}=0$.

From (1) and (3) of Proposition 5.1 we obtain

Proposition 6.8. If $K$ is a tensor field on $M$ and $X$ is a vector field on $M$ sutisfying $\mathcal{L}_{X} K=0$, then

$$
\mathcal{L}_{X^{C}}\left(K^{C}\right)=0 \quad \text { and } \quad \mathcal{L}_{X^{V}}\left(K^{C}\right)=0 .
$$

In particular, we have

Proposition 6.9. If $X$ is a Killing vector field of a pseudo-Riemannian manifold $M$ with metric $g$, then both $X^{c}$ and $X^{v}$ are Killing vector fields of the pseudo-Riemannian manifold $T(M)$ with metric $g^{C}$.

Going back to a general vector field $X$ on $M$, we shall consider $X^{c}$ a little more geometrically. Let $\exp t X$ be a local 1-parameter group of transformations of $M$ generated by $X$. From the coordinate expression of $X^{c}$ it is not hard to see that the induced local 1-parameter group (exp $t X)_{*}$ of transformations of $T(M)$ (where $(\exp t X)_{*}$ denotes the differential of $\exp t X$ for each fixed $t$ ) coincides with a local 1-parameter group exp $t X^{c}$ of transformations of $T(M)$. Hence we have 
Proposition 6.10. If a vector field $X$ on $M$ is complete in the sense that it generates a global 1-barameter group of transformations of $M$, then $X^{c}$ is also a complete vector field on $T(M)$.

REMARK. From the coordinate expression for $X^{v}$ we see immediately that $X^{V}$ is complete whether $X$ is complete or not.

From Propositions 6.9 and 6.10 and the remark above we obtain

Proposition 6.11. If $M$ is a homogeneous pseudo-Riemannian manifold with metric $g$, so is $T(M)$ with metric $g^{C}$.

Similarly we have

Proposition 6.12. If $M$ is a homogeneous (almost) complex manifold with (almost) complex structure $J$, so is $T(M)$ with (almost) complex structure $J^{c}$.

\section{Complete lifts of affine connections.}

Let $\nabla$ be the covariant differentiation defined by an affine connection of $M$. Then there exists a unique affine connection of $T(M)$ whose covariant differentiation $\nabla^{C}$ satisfies

$$
\nabla_{X}^{C} C\left(Y^{C}\right)=\left(\nabla_{X} Y\right)^{C} \quad \text { for } \quad X, Y \in \mathscr{I}_{0}^{1}(M) .
$$

Our assertion may be verified by a simple calculation using connection components. Let $\Gamma_{j k}^{i}$ be the connection components for $\nabla$ with respect to a local coordinate system $x^{1}, \cdots, x^{n}$. With respect to the induced local coordinate system $x^{1}, \cdots, x^{n}, y^{1}, \cdots, y^{n}$ of $T(M)$, we set

$$
\begin{gathered}
\tilde{\Gamma}_{j k}^{i}=\Gamma_{j k}^{i}, \quad \tilde{\Gamma}_{j \bar{k}}^{i}=0, \quad \tilde{\Gamma}_{\bar{j} k}^{i}=0, \quad \tilde{\Gamma}_{\bar{j} \bar{k}}^{i}=0, \\
\tilde{\Gamma}_{j k}^{\bar{i}}=\frac{\partial \Gamma_{j k}^{i}}{\partial x^{l}} y^{l}, \quad \tilde{\Gamma}_{j \bar{k}}^{\bar{i}}=\Gamma_{j k}^{i}, \quad \tilde{\Gamma}_{\bar{j} k}^{\bar{i}}=\Gamma_{j k}^{i}, \quad \tilde{\Gamma}_{j \overline{j k}}^{\bar{i}}=0,
\end{gathered}
$$

where the indices with bars refer to $y^{1}, \cdots, y^{n}$. Then the $\tilde{\Gamma}$ 's are the connection components for $\nabla^{C}$.

PROPOSITION 7.1. If $T$ and $R$ are the torsion and the curvature tensor fields for $\nabla$, then $T^{c}$ and $R^{C}$ are the torsion and the curvature tensor fields for $\nabla^{C}$.

PRoof. Our proposition follows from the following formulas:

$$
\begin{aligned}
& T^{C}\left(X^{C}, Y^{C}\right)=(T(X, Y))^{C}=\left(\nabla_{X} Y-\nabla_{Y} X-[X, Y]\right)^{C} \\
& =\nabla_{X}^{C} Y^{C}-\nabla_{Y^{C}}^{C} X^{C}-\left[X^{C}, Y^{C}\right] . \\
& \begin{aligned}
R^{C}\left(X^{C}, Y^{C}\right) Z^{C}=(R(X, Y) Z)^{C} & =\left(\left[\nabla_{X}, \nabla_{Y}\right] Z-\nabla_{[X, Y]} Z\right)^{C} \\
& =\left[\nabla_{X^{C}}^{C}, \nabla_{Y^{C}}^{C}\right] Z^{C}-\nabla_{\left[X^{C}, Y^{C}\right]} Z^{C} .
\end{aligned}
\end{aligned}
$$

Q.E. D.

Proposition 7.2. For any tensor field $K$ on $M$ and any vector field $X$ on $M$, we have

$$
\nabla_{X}^{C} C\left(K^{C}\right)=\left(\nabla_{X} K\right)^{C}
$$


Proof. As usual it suffices to verify these formulas in the special cases where $K=f \in \mathscr{I}_{0}^{0}(M), K=d f \in \mathscr{I}_{1}^{0}(M)$ and $K=Y \in \mathscr{I}_{0}^{1}(M)$.

(1). If $K=f$, then $\nabla_{X^{C}}^{C}\left(f^{c}\right)=X^{C} f^{C}=\mathcal{L}_{X^{c}} f^{C}=\left(\mathcal{L}_{X} f\right)^{C}=\left(\nabla_{X} f\right)^{c}$. If $K=Y$, then the formula to be verified is nothing but the definition of $\nabla^{c}$. If $K=d f$ or more generally $K=\omega \in \mathscr{T}_{1}^{0}(M)$, then

$$
\begin{aligned}
\left(\nabla_{X}^{C} C \omega^{C}\right)\left(Y^{C}\right) & =\nabla_{X}^{C} C\left(\omega^{C}\left(Y^{C}\right)\right)-\omega^{C}\left(\nabla_{X}^{C} Y^{C}\right)=\nabla_{X}^{C} C(\omega(Y))^{C}-\omega^{C}\left(\left(\nabla_{X} Y\right)^{C}\right) \\
& =\left(\nabla_{X}(\omega(Y))\right)^{C}-\left(\omega\left(\nabla_{X} Y\right)\right)^{C}=\left(\left(\nabla_{X} \omega\right)(Y)\right)^{C} \\
& =\left(\nabla_{X} \omega\right)^{C}\left(Y^{C}\right) .
\end{aligned}
$$

Hence,

$$
\nabla_{X}^{C} C \omega^{C}=\left(\nabla_{X} \omega\right)^{C} .
$$

(2). This follows from (1) and Proposition 5.1 as follows:

and hence

$$
\gamma_{X} C\left(\nabla^{c} K^{c}\right)=\nabla_{X}^{C}\left(K^{c}\right)=\left(\nabla_{X} K\right)^{C}=\left(\gamma_{X} \nabla K\right)^{c}=\gamma_{X} C(\nabla K)^{C}
$$

$$
\nabla^{C} K^{C}=(\nabla K)^{C} .
$$

(3). If $K=f$, then by Proposition 5.1 we have

$$
\nabla_{X}^{C}\left(f^{v}\right)=\mathcal{L}_{X^{C}}\left(f^{V}\right)=\left(\mathcal{L}_{X} f\right)^{V}=\left(\nabla_{X} f\right)^{V} .
$$

If $K=Y$, then write $X=\xi^{i}\left(\partial / \partial x^{i}\right)$ and $Y=\eta^{i}\left(\partial / \partial x^{i}\right)$. Then

$$
\begin{aligned}
\nabla_{X}^{C}\left(Y^{V}\right) & =\left(\frac{\partial \eta^{i}}{\partial x^{j}} \xi^{j}+\tilde{\Gamma}_{j \bar{k}}^{\bar{i}} \xi^{j} \eta^{k}\right) \frac{\partial}{\partial y^{i}} \\
& =\left(\frac{\partial \eta^{i}}{\partial x^{j}} \xi^{j}+\Gamma_{j k}^{i} \xi^{j} \eta^{k}\right) \frac{\partial}{\partial y^{i}} \\
& =\left(\nabla_{X} Y\right)^{V} .
\end{aligned}
$$

Either by a similar calculation using a local coordinate system or by a calculation similar to the one in the proof of (1), we obtain

$$
\nabla_{X}^{C} C\left(\omega^{V}\right)=\left(\nabla_{X} \omega\right)^{V} \quad \text { for every } \omega \in \mathscr{I}_{1}^{0}(M) .
$$

(4). The proof is similar to that for (2).

(5). This may be proved in the same way as (3). Or (7) of Proposition 5.1 and (2) may be used as follows: 


$$
\nabla_{X}^{C} V\left(K^{c}\right)=\gamma_{X} v\left(\nabla^{c} K^{c}\right)=\gamma_{X} v\left((\nabla K)^{C}\right)=\left(\gamma_{X} \nabla K\right)^{v}=\left(\nabla_{X} K\right)^{v}
$$

(6). The proof is similar to that of (5).

Q.E.D.

Proposition 7.3. Let $M$ be a manifold with affine connection $\nabla$. Then a Jacobi field along a geodesic of $M$, considered as a curve in $T(M)$, is a geodesic of $T(M)$ with respect to the affine connection $\nabla^{C}$, and vice versa.

PROOF. From the coordinate expression for the equation of a geodesic, we know that the geodesics of $M(\operatorname{resp} .(T(M))$ are determined by the symmetric part of the connection components of $\nabla$ (resp. $\left.\nabla^{C}\right)$. We may therefore assume without loss of generality that $\nabla$ (and hence also $\nabla^{C}$ ) is torsionfree. Let $x^{1}, \cdots, x^{n}, y^{1}, \cdots, y^{n}$ be the local coordinate system in $T(M)$ induced by a local coordinate system $x^{1}, \cdots, x^{n}$ of $M$. Then a geodesic of $T(M)$ is given by the following set of equations.

$$
\begin{gathered}
\frac{d^{2} x^{i}}{d t^{2}}+\Gamma_{j k}^{i} \frac{d x^{j}}{d t}-\frac{d x^{k}}{d t}=0, \\
\frac{d^{2} y^{i}}{d t^{2}}+-\frac{\partial \Gamma_{j k}^{i} y^{l}}{\partial x^{l}} \frac{d x^{j}}{d t} \frac{d x^{k}}{d t}+2 \Gamma_{j k}^{i} \frac{d y^{j}}{d t} \frac{d x^{k}}{d t}=0 .
\end{gathered}
$$

From (1) we see that a geodesic of $T(M)$ projects upon a geodesic of $M$. We transform (2) as follows.

$$
\begin{aligned}
& \frac{d}{d t}\left(\frac{d y^{2}}{d t}+\Gamma_{i k}^{i} \frac{d x^{j}}{d t} y^{k}\right)+\Gamma_{l h}^{i} \frac{d x^{t}}{d t}\left(\frac{d y^{h}}{d t}+\Gamma_{j k}^{i} \frac{d x^{j}}{d t} y^{k}\right) \\
& +\left(\frac{\partial \Gamma_{j k}^{i}}{\partial x^{l}}-\frac{\partial \Gamma_{j l}^{i}}{\partial x^{k}}+\Gamma_{l h}^{i} \Gamma_{k j}^{h}-\Gamma_{k h}^{i} \Gamma_{l j}^{h}\right) y^{l} \frac{d x^{j}}{d t} \frac{d x^{k}}{d t}=0 .
\end{aligned}
$$

If we denote by $\frac{\delta}{d t}$ the covariant differentiation in $t$, then (3) may be written as follows.

$$
\frac{\delta^{2} y^{2}}{d t^{2}}+R_{j l k}^{i} y^{l} \frac{d x^{j}}{d t} \frac{d x^{k}}{d t}=0 .
$$

This shows that $y^{i}(t)$ is a Jacobi field along the geodesic $x^{i}(t)$ of $M$. Q.E.D.

As an immediate consequence, we have

Proposition 7.4. If $M$ is complete with respect to an affine conneciion $\nabla$. then $T(M)$ is complete with respect to $\nabla^{C}$, and vice versa.

The following result relates the complete lift of an affine connection with Proposition 6.3.

Proposition 7.5. If $\nabla$ is the Riemannian connection of $M$ with respect to a pseudo-Riemannian metric $g$, then $\nabla^{C}$ is the Riemannian connection of $T(M)$ with respect to the pseudo-Riemannian metric $g^{c}$.

PROOF. Since the Riemannian connection is a unique torsionfree connection for which the metric is parallel, our proposition follows from Proposition 
7.1 and (2) of Proposition 7.2 applied to $K=g$.

Q. E. D.

Proposition 7.6. Let $\nabla$ be an affine connection on $M$. If $X$ is an infinitesimal affine transformation of $M$, then both $X^{c}$ and $X^{v}$ are infinitesimal affine transformations of $T(M)$ with respect to $\nabla^{C}$.

Proof. A necessary and sufficient condition for $X$ to be an infinitesimal affine transformation of $M$ is that

$$
\mathcal{L}_{X} \circ \nabla_{Y}-\nabla_{Y} \circ \mathcal{L}_{X}=\nabla_{[X, Y]} \quad \text { for every } \quad Y \in \mathscr{I}_{0}^{1}(M) .
$$

Making use of Propositions 5.1 and 7.2 we verify easily

$$
\mathcal{L}_{\widetilde{X}} \circ \nabla_{\widetilde{Y}}^{C}-\nabla_{\widetilde{Y}}^{C} \circ \mathcal{L}_{\widetilde{X}}=\nabla_{[\widetilde{X}, Y]}^{C}
$$

in the following special cases: $\tilde{X}=X^{c}$ or $=X^{v}$ and $\tilde{Y}=Y^{c}$ or $=Y^{v}$. From the coordinate expressions for $Y^{c}$ and $Y^{v}$ we see that the formula above is valid for an arbitrary $\tilde{Y}$. This proves that both $X^{C}$ and $X^{v}$ are infinitesimal affine transformations of $\nabla^{C}$.

Q.E. D.

From Propositions 6.10 and 7.6 we obtain

Proposition 7.7. If the group of affine transformations of $M$ with $\nabla$ is transitive on $M$, then the group of affine transformations of $T(M)$ with respect to $\nabla^{C}$ is transitive on $T(M)$.

From Propositions 7.1 and 7.2 we obtain

Proposition 7.8. Let $T$ and $R$ be the torsion and the curvature tensor fields of an affine connection $\nabla$. of $M$. According as $T=0, \nabla T=0, R=0$ or $\nabla R=0$, we have $T^{C}=0, \nabla^{C} T^{C}=0, R^{C}=0$ or $\nabla^{C} R^{C}=0$. In particular, if $M$ is locally affine symmetric with respect to $\nabla$, so is $T(M)$ with respect to $\nabla^{C}$.

From Propositions 7.5 and 7.8 we obtain

Proposition 7.9. If $M$ is locally pseudo-Riemannian symmetric with respect t) $g$, so is $T(M)$ with respect to $g^{C}$.

REMARK. A pseudo-Riemannian manifold $M$ with metric $g$ is said to be of constant curvature $k$ if

$$
R(X, Y) Z=k(g(Z, Y) X-g(Z, X) Y) \quad \text { for } \quad X, Y, Z \in \mathscr{I}_{0}^{1}(M)
$$

If $M$ is of constant curvature $k$, then the curvature $R^{C}$ of $g^{C}$ may be calculated as follows.

$$
\begin{aligned}
& R^{C}\left(X^{c}, Y^{c}\right) Z^{c}=(R(X, Y) Z)^{c}=k(g(Z, Y) X-g(Z, X) Y)^{c} \\
& =k\left((g(Z, Y))^{c} X^{v}+(g(Z, Y))^{v} X^{c}-(g(Z, X))^{c} Y^{v}-(g(Z, X))^{v} Y^{c}\right) \\
& =k\left(g^{C}\left(Z^{c}, Y^{C}\right) X^{v}+g^{v}\left(Z^{c}, Y^{C}\right) X^{C}-g^{C}\left(Z^{C}, X^{C}\right) Y^{v}-g^{V}\left(Z^{C}, X^{C}\right) Y^{C}\right) .
\end{aligned}
$$

Define a tensor field $E$ of type $(1,1)$ on $T(M)$ by

$$
E=\frac{\partial}{\partial y^{i}} \otimes d x^{2}
$$


in terms of the local coordinate system $x^{1}, \cdots, x^{n}, y^{1}, \cdots, y^{n}$ induced by a local coordinate system $x^{1}, \cdots, x^{n}$ of $M$. ( $E$ may be defined without local coordinate systems.) Then $E X^{C}=X^{V}$ and $g^{V}(*, E *)=g^{C}(*, *)$. Hence

$$
\begin{aligned}
R^{c}\left(X^{c}, Y^{c}\right) Z^{c}= & k\left(g^{c}\left(Z^{c}, Y^{c}\right) E X^{c}+g^{c}\left(Z^{c}, E Y^{c}\right) X^{c}\right. \\
& \left.-g^{c}\left(Z^{c}, X^{c}\right) E Y^{c}-g^{c}\left(Z, E X^{c}\right) Y^{c}\right) .
\end{aligned}
$$

Using this formula it is not hard to prove that the complete lift $g^{c}$ of a metric $g$ of constant curvature $k$ has constant curvature if and only if $k=0$. Similarly, the complete lift $g^{C}$ of an Einstein metric $g$ is again an Einstein metric if and only if $g$ has vanishing Ricci tensor.

The operations such as $\mathcal{L}_{X}, \alpha_{X}, \alpha$, vertical lift and complete lift are all natural in the sense that they commute with any diffeomorphism of one manifold onto another. In particular, if $h$ is a transformation of $M$ leaving a connection $\nabla$ invariant, then its differential $h_{*}: T(M) \rightarrow T(M)$ leaves the connection $\nabla^{C}$ invariant. Similarly, if $h$ leaves a tensor field $K$ on $M$ invariant, then $h_{*}$ leaves $K^{C}$ invariant. Applying this reasoning to a pseudo-Riemannian (or affine) symmetric space $M$ and its symmetries, we obtain

Proposition 7.10. If $M$ is a pseudo-Riemannian (resp. affine) symmetric space with metric $g$ (resp. connection $\nabla$ ), then $T(M)$ is also a pseudo-Riemannian (resp. affine) symmetric space with metric $g^{C}$ (resp. connection $\nabla^{C}$ ).

REMARK. This proposition is related to the following known result, [6]. If $G / H$ is a reducible affine symmetric space with $G$ simple, then it is a tangent bundle over a compact Riemannian symmetric space. See also [1], [4] for fibrations of affine symmetric spaces.

We shall make a concluding remark about generalizations of results in this paper. One possible generalization is, as we have already pointed out in the introduction, to lift tensor fields and connections to the bundle $A(M)$ defined by a local algebra $A$. Another generalization is to lift a wider class of geometric structures of $M$ to the tangent bundle $T(M)$. For instance, would it be possible to associate with each $G$-structure on $M$ a naturally induced $G^{\prime}$-structure on $T(M)$, where $G^{\prime}$ is a certain subgroup of $G L(2 n ; R)$ ? We have seen that a pseudo-Riemannian structure on $M$ gives rise to a pseudo-Riemannian structure on $T(M)$. Moreover, $T(M)$ carries an obvious $n$-dimensional distribution. This means that for $G=O(n)$, a correct $G^{\prime}$ may not be $S O(n, n)$ but a certain subgroup of $S O(n, n)$. Similar comments may apply to almost complex structures, symplectic structures, etc.. It seems that a conformal structure on $M$ does not immediately induce a conformal structure on $T(M)$. The question of associating a correct $G^{\prime}$-structure on $T(M)$ to a $G$-structure on $M$ is probably related to the following question on holonomy: how is the holonomy group of $T(M)$ with $\nabla^{C}$ related to the holonomy 
of $M$ with $\nabla$. Another related question is this. In [2] it was shown that if $P$ is a principal fibre bundle over $M$ with group $G$ and if $\Gamma$ is a connection in $P$, then there exists a naturally induced connection $T(\Gamma)$ in the bundle $T(P)$ over $T(M)$ with group $T(G)$ and the restricted holonomy group $\Phi^{0}(T(\Gamma))$ of $T(\Gamma)$ is isomorphic in a natural way with $T\left(\Phi^{0}(\Gamma)\right)$, where $\Phi^{0}(\Gamma)$ is the restricted holonomy group of $\Gamma$. (The last statement is essentially the socalled Holonomy Theorem of Ambrose-Singer.) If $P$ is the bundle $L(M)$ of linear frames of $M$, then the connection $T(\Gamma)$ seems to be related to the complete lift of $\Gamma$.

\author{
Tokyo Institute of Technology \\ and \\ University of California, Berkeley
}

\title{
Bibliography
}

[1] M. Berger, Les espaces sy métriques non compacts, Ann. Sci. Ecole Norm., Sup., (3) 74 (1957), 85-177.

[2] S. Kobayashi, Theory of connections, Ann. Mat. Pura Appl., 43 (1957), 119-194.

[3] S. Kobayashi and K. Nomizu, Foundations of differential geometry, Interscience Tract No. 15 (1963).

[4] S. Koh, On affine symmetric spaces, Trans. Amer. Math. Soc., 119 (1965), 291309.

[5] A. J. Ledger and K. Yano, The tangent bundle of a locally symmetric space, J. London Math. Soc., 40 (1965), 487-492.

[6] T. Nagano, Transformation groups on symmetric spaces, to appear in Trans. Amer. Math. Soc..

[7] S. Sasaki, On the differential geometry of tangent bundles of Riemannian manifolds, Tôhoku Math. J., 10 (1958), 338-354.

[8] S. Tanno, An almost complex structure of the tangent bundle of an almost contact manifold, Tôhoku Math. J., 17 (1965), 7-15.

[9] P. Tondeur, Structure presque kählérienne naturelle sur la fibré des vecteurs covariants d'une variété riemannienne, C. R. Acad. Sci. Paris, 254 (1962), 407-408.

[10] A. Weil, Théorie des points proches sur les variétés différentiables, Colloque de géométrie différentielle, Strasbourg, (1953), 111-117.

[11] K. Yano, The theory of Lie derivatives and its applications, North Holland Pub. Co., Amsterdam, 1957.

[12] K. Yano, On a structure $f$ satisfying $f^{3}+f=0$, Technical Report, University of Washington, 1961.

[13] K. Yano, On a structure defined by a tensor field $f$ of type $(1,1)$ satisfying $f^{3}+f=0$, Tensor, 14 (1963), 99-109.

[14] K. Yano and E.T. Davies, On the tangent bundle of Finsler and Riemannian manifolds, Rend. Circ. Mat. Palermo, 12 (1963), 211-228.

[15] K. Yano and A. J. Ledger, Linear connections on tangent bundles, J. London Math. Soc., 39 (1964), 495-500. 